\title{
Methodology for development of the Chinese evidence-based Clinical Practice Guideline of the Diagnosis and Management of Cough
}

\author{
Mei Jiang, Yan-Qing Xie, Jia-Xing Xie, Xiang-Wei Zou, Ke-Fang Lai \\ State Key Laboratory of Respiratory Disease, National Clinical Research Center for Respiratory Disease, Guangzhou Institute of Respiratory \\ Health, First Affiliated Hospital of Guangzhou Medical University, Guangzhou 510120, China \\ Correspondence to: Ke-Fang Lai, PhD. State Key Laboratory of Respiratory Disease, National Clinical Research Center for Respiratory Disease, \\ Guangzhou Institute of Respiratory Health, First Affiliated Hospital of Guangzhou Medical University, 151 Yanjiang Road, Guangzhou 510120, \\ China. Email: klai@163.com. \\ Comment on: Lai K, Shen H, Zhou X, et al. Clinical Practice Guidelines for Diagnosis and Management of Cough-Chinese Thoracic Society (CTS) \\ Asthma Consortium. J Thorac Dis 2018;10:6314-51.
}

Submitted Apr 20, 2018. Accepted for publication Sep 12, 2018.

doi: 10.21037/jtd.2018.09.154

View this article at: http://dx.doi.org/10.21037/jtd.2018.09.154

Cough is one of the most common symptom for which patients seek medical care $(1,2)$ and might frequently lead to impaired quality of life (3), absenteeism, social embarrassment and other adverse effects such as urinary incontinence (4). Chronic cough is often misdiagnosed because of no overt chest radiographic findings (5-7). This results in unnecessary repetitive testing and inappropriate treatment in China, such as the overuse of antibiotics, which translates into limited clinical improvement but could lead to potential adverse side effects and constitute a significant healthcare burden (5-8).

Because cough is a global problem managed by a variety of disciplines (3), assessment and management of cough remain the major challenge for clinicians and vary significantly in practice (9). It was important to identify and implement evidence-based strategies to ensure the quality of healthcare for patients with cough (3,9-12). To standardize clinical practice and improve treatment outcomes in China, the Panel of Chinese Thoracic Society (CTS) Asthma Consortium drafted the first edition of the Chinese Guidelines for Diagnosis and Treatment of Cough (Draft) published in 2005 (13) and the updated edition in $2009(6,7)$. Since the release of the Chinese Cough Guidelines, the management of cough in China has been improved. However, the Chinese Cough Guideline was developed by non-evidence-based methods and has not been updated since 2009. Recently, there have been significant advances in cough research and increased understanding of the pathogenesis, etiology, diagnosis, and management of cough. To further refine the guideline and include the latest evidence, in 2014 the CTS Asthma Consortium initiated a task force to revise the Chinese Cough Guideline (14).

Rigorous and transparent methods were necessary to develop guidelines (9-12). To ensure that the Chinese Cough Guideline met international and national standards, a modification form by the GRADE (Grading of Recommendations, Assessment, Development, and Evaluation) approach $(15,16)$ for guideline for development was used. It should be helpful and interesting to readers to brief the development and methodology of the latest updated Chinese Evidence-based Cough Guideline, which addresses the best practice in care delivery for patients with cough in China.

\section{Panel selection and composition}

CTS Asthma Consortium convened multidisciplinary panel members. Members of the guideline panel included the established specialists in the relevant clinical or research areas in China, such as respiratory medicine, ear-nose and throat, pediatrics, gastroenterology, Traditional Chinese Medicine (TCM), pharmacology, nursing, the evidencebased medicine, guideline methodology, the clinical epidemiologists, and medical editors. A chair who is a leader in cough research and practice was nominated as being 
responsible for guideline development. The guideline panel was divided into several groups according to different topics, including methodology group. Each group is made up of a director and relevant members clear their roles. All panel members participated in the entire guideline development process via teleconferences, electronic communication and face-to-face conferences. Participants were asked to sign a written statement declaring any Conflict of Interest form (COI) associated with guideline development. Only the members without COIs have voting right when the panel experts made the final recommendations.

\section{Planning guideline}

Specific guideline proposals were made by the guideline panel before guideline development (17), including the following contents: scoping the guideline, targeting the study population, the roles of all guideline development group members, how the guideline will be developed, timeline, funding and budget, predicting the problems which might occur during guideline development and the possible resolutions.

\section{Methodological training}

To guarantee the quality of guideline development, evidence-based medicine and guideline development experts must offer the other guideline panels members adequate training in methodology, including guideline development methodology, literature retrieval, meta-analysis and GRADE approach, participate in the whole guideline development process and solve related problems.

\section{Specifying Key clinical questions}

Clinical specialists in the field were surveyed to identify prioritized topics to address within the scope of the guideline before the planning conference by email. The final critical clinical questions and outcomes were discussed and identified during the first face-to-face experts meeting, and the guideline panel was invited to frame the clinical questions in the PICO (patient-intervention-comparatoroutcome) format.

\section{Evidence retrieval}

The guideline panel retrieves evidence to address the final guideline questions by an exhaustive search of PubMed/
Medline, Embase, and Cochrane Library and four major Chinese academic databases [Chinese Biomedical Literature database (CBM), China National Knowledge Infrastructure (CNKI), VIP database and WANFANG database] up to June 30, 2015 and reference lists of retrieved papers. Two trained and independent groups conducted the literature search for each specific clinical issue according to the inclusion and exclusion criteria and extracted the relevant data. An appraisal of the literature using a specifically designed form was performed. Respiratory physicians conducted the preliminary evaluation of the literature. In cases where consensus could not be obtained due to difficulty in literature appraisal, a meeting of the guideline panel was held for critical review and reappraisal. If necessary, the literature search and evaluation were performed again. Systematic reviews of published literature were performed to retrieve evidence on the benefits and harms of each intervention, patients' preferences, acceptability, feasibility, costs and resource use.

\section{Evidence synthesis and quality grading}

A composite grading system for assessing the quality of evidence and grading recommendation was adopted which combined the grading system recommended by the American College of Chest Physicians (ACCP) Guidelines for Diagnosis and Management of Cough (18) published in 2006 and GRADE (grading of recommendations assessment, development, and evaluation) approach (16) (Table 1). The level of evidence was graded as four categories: high, moderate, low, and very low, and the strength of recommendation was defined as strong, weak and no specific recommendation.

The quality assessment of the body of evidence was based on the GRADE approach. Evidence based on randomized controlled trials began with high-quality evidence, but our confidence in the evidence might be decreased for five reasons (study limitations, inconsistency of results, indirectness of evidence, imprecision and publication bias).

Evidence based on observational studies start with a "low quality" rating, grading upwards may be warranted for three factors (large magnitude effect, dose-response gradient, or plausible confounding which could reduce a demonstrated effect). The quality of evidence across different outcomes as that associated with the critical outcome with the lowest quality evidence. If the evidence originated from systemic reviews/meta-analyses, the AMSTAR (A MeaSurement Tool to assess Systemic Reviews) instrument (19) was used for 
Table 1 Components used in the grading of the quality of evidence and the strength of recommendations

\begin{tabular}{lll}
\hline $\begin{array}{l}\text { Category } \\
\text { Quality of evidence }\end{array}$ & A & Evade \\
& B & $\begin{array}{l}\text { Evidence from high-quality randomized controlled trial (RCT) or systemic reviews/meta-analyses } \\
\text { observational studies }\end{array}$ \\
& C & Evidence from non-randomized, case-control, or other observational studies \\
& D & Expert individual opinion \\
Strength of recommendation & 1 & Strong recommendation \\
& 2 & Weak recommendation \\
& 3 & No specific recommendation (insufficient evidence on which to formulate a recommendation) \\
\hline
\end{tabular}

assessment. Only Systemic reviews/meta-analyses fulfilling nine or more of the eleven criteria were regarded as high quality systemic reviews/meta-analyses.

\section{Formulating recommendations}

A face-to-face conference of the guideline panel was held to formulate the final recommendations. GRADE's Evidence to Decision frameworks was used to guide a structured consensus process and transparent document all decisions made during the meeting. The direction and strength of recommendations are determined by all of the evaluation, including the final level for quality of evidence, benefits and harms, patient's values and preferences and use of resource. Additional consideration related to the research, implementation and monitoring was also discussed. The guideline panel discussed at modified nominal group meetings for each issue or intervention. The decision was made by votes using the modified Delphi method (20). The voting procedure for the nominal group meetings guided by the following rules. In areas of continuing disagreement, a recommendation for or against a particular intervention (compared with a specific alternative) required at least $50 \%$ of participants who were in favour of, with less than $20 \%$ preferring the comparator (the options could be judged equally) (20). Failure to meet this criterion resulted in no recommendation. For a recommendation to be graded as strong rather than weak, at least $70 \%$ of participants were required to endorse it as being strong (20). The guideline panel drafted and ultimately created a final unabridged guideline.

\section{External peer review}

After final review of the unabridged guideline by the guideline panel, the guideline was sent to the senior specialists in this field by email. The specialists were invited to review the guideline and submit comments during a month public review period. The guideline panel reviewed all comments, discussed and incorporated them in to the final version of the guideline when it was necessary.

\section{Time to update planning}

CTS Asthma Consortium decided to update the Chinese Cough Guideline every 3-5 years when import new studies are published that might change the current recommendations.

In conclusion, the updated Chinese Cough guideline used a rigorous and transparent methodology based on GRADE approach to produce evidence-based recommendations for clinical practice, which served as the role example and suggestions for developing high-quality, evidence-based guidelines using sound methodological frameworks in China in the future. Such a standard and dedicate procedure in guideline development would inspire generation of more prestigious guideline documents in China, and should also be practically implicative for relevant work in other parts of the world.

\section{Acknowledgements}

Funding: This work was supported by the National Natural Science Foundation of China [Grant No. 81402772].

\section{Footnote}

Conflicts of Interest: The authors have no conflicts of interest 
to declare.

\section{References}

1. Marchant JM, Newcombe PA, Juniper EF, et al. What is the burden of chronic cough for families? Chest 2008;134:303-9.

2. Lai K, Luo W, Zeng G, et al. Diagnosis and treatment of chronic cough in China: an insight into the status quo. Cough 2012;8:4.

3. Irwin RS, French CT, Lewis SZ, et al. Overview of the management of cough: CHEST Guideline and Expert Panel Report. Chest 2014;146:885-9.

4. French CT, Fletcher KE, Irwin RS. Gender differences in health-related quality of life in patients complaining of chronic cough. Chest 2004;125:482-8.

5. Lai K, Pan J, Chen R, et al. Epidemiology of cough in relation to China. Cough 2013;9:18.

6. Asthma Workgroup, Chinese Society, Respiratory, Diseases (CSRD), Chinese Medical, Association. The Chinese national guidelines on diagnosis and management of cough (December 2010). Chin Med J (Engl) 2011;124:3207-19.

7. Consortium CTSCA. Clinical Practice Guidelines for Diagnosis and Management of Cough (2009). Chin J Tuberc Respir Dis 2009;32:407-13.

8. Lai K. Chinese National Guidelines on Diagnosis and Management of Cough: consensus and controversy. J Thorac Dis 2014;6:S683-8.

9. Jiang M, Guan WJ, Fang ZF, et al. A Critical Review of the Quality of Cough Clinical Practice Guidelines. Chest 2016;150:777-88.

10. Grol R, Cluzeau FA, Burgers JS. Clinical practice guidelines: towards better quality guidelines and increased international collaboration. Br J Cancer 2003;89 Suppl

Cite this article as: Jiang M, Xie YQ, Xie JX, Zou XW, Lai KF. Methodology for development of the Chinese evidence-based Clinical Practice Guideline of the Diagnosis and Management of Cough. J Thorac Dis 2018;10(11):6310-6313. doi: 10.21037/ jtd.2018.09.154
1:S4-8,

11. Lewis SZ, Diekemper R, Ornelas J, et al. Methodologies for the development of CHEST guidelines and expert panel reports. Chest 2014;146:182-92.

12. Lewis SZ, Diekemper RL, French CT, et al. Methodologies for the development of the management of cough: CHEST guideline and expert panel report. Chest 2014;146:1395-402.

13. Consortium CTSCA. Clinical Practice Guidelines for Diagnosis and Management of Cough (2005). Chin J Tuberc Respir Dis 2005;28:738-44.

14. Consortium CTSCA. Clinical Practice Guidelines for Diagnosis and Management of Cough (2015). Chin J Tuberc Respir Dis 2016;39:323-54.

15. Guyatt GH, Oxman AD, Vist GE, et al. GRADE: an emerging consensus on rating quality of evidence and strength of recommendations. BMJ 2008;336:924-6.

16. Guyatt GH, Oxman AD, Kunz R, et al. Going from evidence to recommendations. BMJ 2008;336:1049-51.

17. Organization WH. WHO Handbook of Guideline Development. Available online: http://apps.who.int/ iris/bitstream/10665/75146/1/9789241548441_eng. pdf?ua $=1 \&$ ua $=1$

18. Irwin RS, Baumann MH, Bolser DC, et al. Diagnosis and management of cough executive summary: ACCP evidence-based clinical practice guidelines. Chest 2006;129:1S-23S.

19. Shea BJ, Hamel C, Wells GA, et al. AMSTAR is a reliable and valid measurement tool to assess the methodological quality of systematic reviews. J Clin Epidemiol 2009;62:1013-20.

20. Jaeschke R, Guyatt GH, Dellinger P, et al. Use of GRADE grid to reach decisions on clinical practice guidelines when consensus is elusive. BMJ 2008;337:a744. 